\title{
ANÁLISE DA ENTROPIA DE KOLMOGOROV A PARTIR DE MEDIÇÕES PIV NO ESCOAMENTO DA FASE LÍQUIDA DE UMA COLUNA DE BOLHAS
}

\author{
H. L. MOURA ${ }^{1 *}$, R. L. AMARAL ${ }^{1}$, D. I. S. FORERO ${ }^{1}$, G. J. CASTILHO ${ }^{1}$, S.S.V. VIANNA ${ }^{1}$, M. \\ MORI $^{1}$ \\ ${ }^{1}$ Universidade Estadual de Campinas, Faculdade de Engenharia Química \\ *e-mail: helder_cdc@hotmail.com
}

\begin{abstract}
RESUMO
Devido à sua construção simples e facilidade de operação, colunas de bolhas são frequentemente aplicadas em processos industriais. O conhecimento do comportamento fluidodinâmico desses reatores é importante para o controle da transferência de calor, transferência de massa e taxa de reação química. Muitos trabalhos baseiam-se na análise de flutuações de pressão para descrever o comportamento complexo de uma coluna de bolhas. Porém, sendo uma medida indireta da dinâmica do escoamento, essa torna-se a sua principal desvantagem. Nesse contexto, destaca-se a técnica de velocimetria por imagem de partícula (PIV). A PIV é uma técnica de medida não intrusiva que determina a distribuição de velocidade em uma área de investigação. Este trabalho tem como objetivo avaliar o escoamento da fase líquida usando a análise de caos a partir de medições PIV de flutuações de velocidade em uma seção de coluna de bolhas para diferentes vazões de gás. Para isto, utilizou-se os conceitos da entropia de Kolmogorov. A análise caótica se desponta como um método promissor, tendo como principal vantagem a sensibilidade a pequenas alterações do escoamento, em relação as análises no domínio do tempo e frequência. Os resultados obtidos mostram que é possível extrair informações relevantes a partir das medidas PIV em conjunto com a análise da entropia de Kolmogorov. Contudo, foi observado que para altas vazões a descrição da dinâmica do sistema requer maior atenção.
\end{abstract}

\section{INTRODUÇÃO}

Colunas de bolhas têm algumas características que as tornam adequadas para aplicações industriais. São amplamente utilizadas em indústrias químicas e petroquímicas, possibilitam boas propriedades de transferência de massa e calor, índice de mistura elevados, design simples e baixo custo operacional e de manutenção (DECKWER e FIELD, 1992; YANG, 2003). Compreender a hidrodinâmica das colunas de bolhas e as transições dos regimes são de grande importância para o projeto, operação, controle e scale-up (FAN, 1989). Uma vez que os mecanismos subjacentes e muitos dos parâmetros de funcionamento podem tornar o comportamento da coluna de bolhas complexo, uma modelagem teórica ou uma abordagem experimental se torna difícil. Esse fato está diretamente atrelado aos movimentos instáveis das numerosas bolhas e as interações não lineares com a parede da própria coluna (FAN, 1989; NEDELTCHEV et al., 2007; ABBASI et al., 2013; LI et al., 2013).

Um grande número de técnicas de medição, incluindo métodos intrusivos e não intrusivos, foram desenvolvidos para investigar o comportamento da dinâmica de uma coluna de bolhas (YANG et al., 2007). Uma revisão abrangente das técnicas de medição em reatores gás-líquido e também 
gás-líquido-sólido pode ser encontrada em Boyer et al. (2002). No que se segue, entre as diferentes técnicas de medição, com ênfase nas técnicas não intrusivas, flutuações de pressão são amplamente utilizadas para caracterizar a hidrodinâmica e transição de regimes em colunas de bolhas (BRIENS e ELLIS, 2005; SHOU e LEU, 2005; NEDELTCHEV et al., 2007; ZAID et al., 2011; ABBASI et al., 2013).

Por outro lado, técnicas ópticas não intrusivas também se despontam como métodos promissores, com destaque para a técnica de velocimetria por imagem de partículas (PIV). A técnica PIV é frequentemente empregada na caracterização do campo de velocidade em diversos tipos de escoamentos multifásicos presentes em colunas de bolhas (BRÖDER e SOMMERFELD, 2007, 2002). Contudo, para garantir uma boa representação da dinâmica destes escoamentos, assim como a confiabilidade dos campos de velocidade, alguns trabalhos foram desenvolvidos: da Costa et al. (2014) estudaram o erro da medida causado pela distorção das bolhas; Sanchez-Forero et al. (2014) analisaram a qualidade da medida PIV com o aumento da vazão de gás em uma coluna de bolhas com expansão de diâmetro; Amaral et al. (2014a) investigaram $o$ efeito da função de mapeamento PIV na distribuição da velocidade da fase líquida na seção de uma coluna de bolhas e Amaral et al. (2014b) avaliaram o efeito da função de mapeamento PIV na distribuição da velocidade da fase líquida na seção de uma coluna de bolhas.

Em relação aos métodos de análise, vários foram propostos e aplicados para séries de flutuações de pressão, como análise estatística (DRAHOS et al., 1990), análise espectral (ABBASI et al., 2013), wavelet (PARK e KIM, 2003) e caos (LETZEL et al., 1997, BRIENS e ELLIS, 2005, NEDELTCHEV et al., 2007 e MOSDORF e WYSZKOWSKI, 2011). Estes métodos podem ser aplicados para caracterização da hidrodinâmica de colunas de bolhas, por exemplo, transição de regime $\mathrm{e}$ comportamento das bolhas (ABBASI et al., 2013).

A descrição da hidrodinâmica em colunas de bolhas, tendo em vista as distintas regiões de contato entre as fases, não é trivial devido à existência de comportamento complexo, não linear, induzido pela interação altamente turbulenta entre as fases gasosa e líquida, principalmente em regimes heterogêneos (LI et al., 2013). Portanto, a análise de caos, a partir de sinais experimentais, oferece uma alternativa para diferenciar um sistema determinístico e ordenado de um sistema estocástico e não ordenado, em relação a métodos tradicionais como, por exemplo, o espectro de potência de Fourier e análise estatística (ABARBANEL, 1996).

A análise de caos utiliza a reconstrução do espaço de fase para mapear a evolução de um processo no tempo, por trajetórias em um espaço de fase multidimensional. Os dados imersos neste espaço de fase podem ter sua complexidade medida a partir da entropia de Kolmogorov. A entropia de Kolmogorov indica a taxa de perda de informação ao longo do atrator ou o grau de previsibilidade de pontos ao longo do atrator com evolução do tempo no sistema (GRASSBERGER e PROCACCIA, 1983). Foi verificado que a entropia de Kolmogorov é um parâmetro extremamente útil para quantificar o grau de caos que as colunas de bolhas aparentemente exibem (LETZEL, et al., 1997; VIAL et al., 2000; LIN et al., 2001; GOURICH et al., 2006; NEDELTCHEV et al., 2007; AJBAR et al., 2009; NEDELTCHEV et al., 2011). Grassberger e Procaccia (1983) relatam que a entropia de Kolmogorov maior que zero é uma condição suficiente para o caos, em que o sistema caótico é apenas previsível em um intervalo de tempo limitado. 
Este trabalho tem como objetivo avaliar o escoamento da fase líquida usando a análise caos a partir de medições PIV das séries temporais de flutuações do módulo de velocidade em uma região central de uma coluna de bolhas para diferentes vazões de gás. $\mathrm{O}$ comportamento complexo da hidrodinâmica subjacente da coluna de bolha foi avaliado a partir da entropia de Kolmogorov.

\subsection{Velocimetria por imagem de partículas (PIV)}

$\mathrm{O}$ princípio de funcionamento da PIV 2D Clássico é ilustrado na Figura 1.

Figura 1 - Princípio de funcionamento da técnica PIV 2D Clássico.
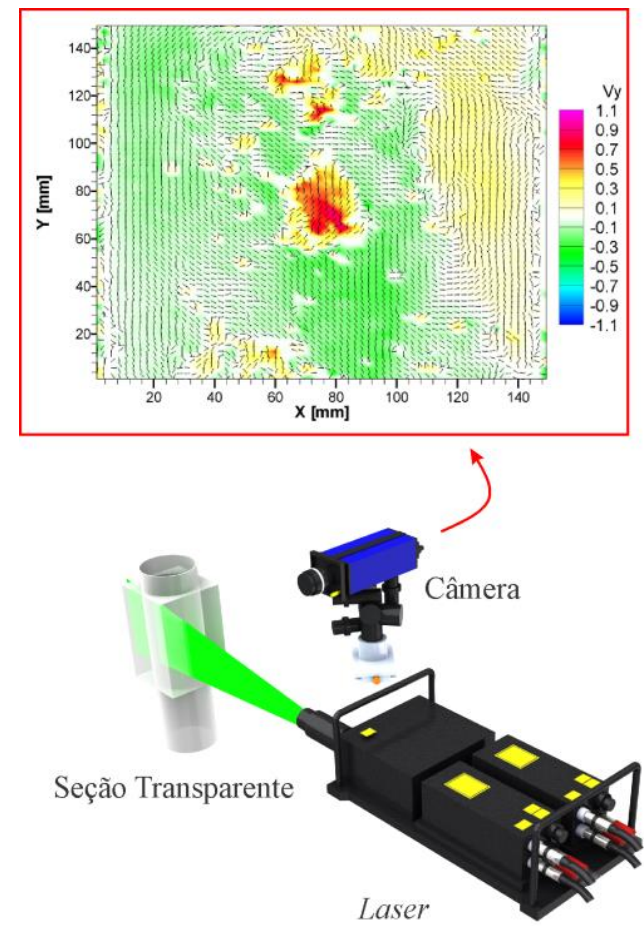

Fonte: Adaptado de AMARAL (2013).

A técnica PIV emprega partículas traçadoras que devem seguir fielmente $o$ escoamento do fluido, para extrair indiretamente a sua velocidade. As partículas são iluminadas por uma folha de luz pulsada e espalham luz em uma lente fotográfica, geralmente localizada a $90^{\circ}$ da folha de luz, de modo que o seu plano do objeto em foco coincida com a fatia iluminada do escoamento. As imagens são formadas em um sensor e são, em seguida, transferidas para um computador para análise (ADRIAN, 1991). Em vez de determinar o deslocamento individual das imagens partículas, é utilizado um algoritmo para correlacionar as imagens de partícula nos dois tempos (gravação double-frame), para determinar o movimento médio de pequenas populações de imagens de partículas contidas em regiões, conhecidas como janelas de interrogação, fornecendo um vetor de deslocamento por janela (PRASAD, 2000). A relação entre a imagem (projeção) e as coordenadas do espaço físico (o objeto) é estabelecida por um procedimento de calibração fornecendo uma função de mapeamento do sistema de imagem.

\subsection{Análise de caos: entropia de Kolmogorov}

Schouten et al. (1994) estimaram a entropia de Kolmogorov em um tempo médio necessário para duas órbitas do atrator, inicialmente muito próximo uns dos outros, divergir. De acordo com Takens (1981) e Grassberger e Procaccia (1983), a separação de pontos vizinhos em órbitas diferentes é assumida ser exponencial. $\mathrm{O}$ intervalo de tempo necessário para que dois pontos inicialmente próximos se separem por uma distância maior do que $l_{0}$ será distribuído exponencialmente de acordo com

$$
C\left(t_{0}\right) \propto e^{-K t_{0}}
$$

A distribuição discreta para uma série temporal amostradas com uma frequência de amostragem $\mathrm{f}_{\mathrm{s}}$ e passo de tempo $\tau_{s}=1 / f_{s}$ é

$C(b) \propto e^{-K b \tau_{s}}$

A função de distribuição cumulativa (Equação 2) descreve a queda exponencial em 
função de b. Esta variável b é igual ao número de pares sequenciais de pontos no atrator, dado um par inicial de pontos independentes dentro de uma distância $l_{0}$, em que a distância entre os pontos é pela primeira vez maior que a distância máxima especificada $1_{0}$. Em outras palavras, b é obtido a partir do número de vezes que

$$
\left\|X_{i+b-1}-X_{j+b-1}\right\| \leq l_{0} \text { com } \mathrm{b}=1,2,3, \ldots
$$

desde

$$
\text { que }
$$$$
\left\|X_{i}-X_{j}\right\| \leq l_{0}
$$

enquanto $\left\|X_{i+b}-X_{j+b}\right\|>l_{0}$.

Um valor de $b_{m}$ é obtido para cada par sequencial i,j investigado. Desse modo, o valor de $\bar{b}$ é calculado pela média dos valores $b_{m}$

$\bar{b}=\frac{1}{M} \sum_{i=1}^{M} b_{m}$

em que $\mathrm{M}$ é o número de pares investigados. Finalmente, a entropia de máxima verossimilhança (maximum likelihood Kolmogorov entropy), $\mathrm{K}_{\mathrm{ML}}$, é estimada como

$$
K \approx K_{M L}=-\frac{1}{\tau_{s}} \ln \left[1-\frac{1}{b}\right]
$$

O desvio padrão $s\left(K_{M L}\right)$ é obtido por

$$
s\left(K_{M L}\right)=\left[\tau_{S} \sqrt{M} K_{M L} \sqrt{\bar{b}(\bar{b}-1)}\right]^{-1}
$$

Em que $\tau_{s}$ é o tempo de amostragem. Para $K_{M L} \tau_{S}<<1 \quad$ o desvio padrão pode ser aproximado por:

$$
s\left(K_{M L}\right) \cong \frac{1}{\sqrt{M}}
$$

\section{MATERIAIS E MÉTODOS}

A Figura 2 apresenta o esquema da coluna de bolhas e do sistema PIV utilizado. O procedimento experimental detalhado é encontrado no trabalho de Amaral et al (2014a).

Figura 2 - (a) Coluna, sistema PIV e caixa com a fenda. (b) Área de investigação. (c) Distribuidor da fase gasosa com 21 furos. (d) Arranjo do sistema PIV na coluna. (e) ponto central r/R =0.

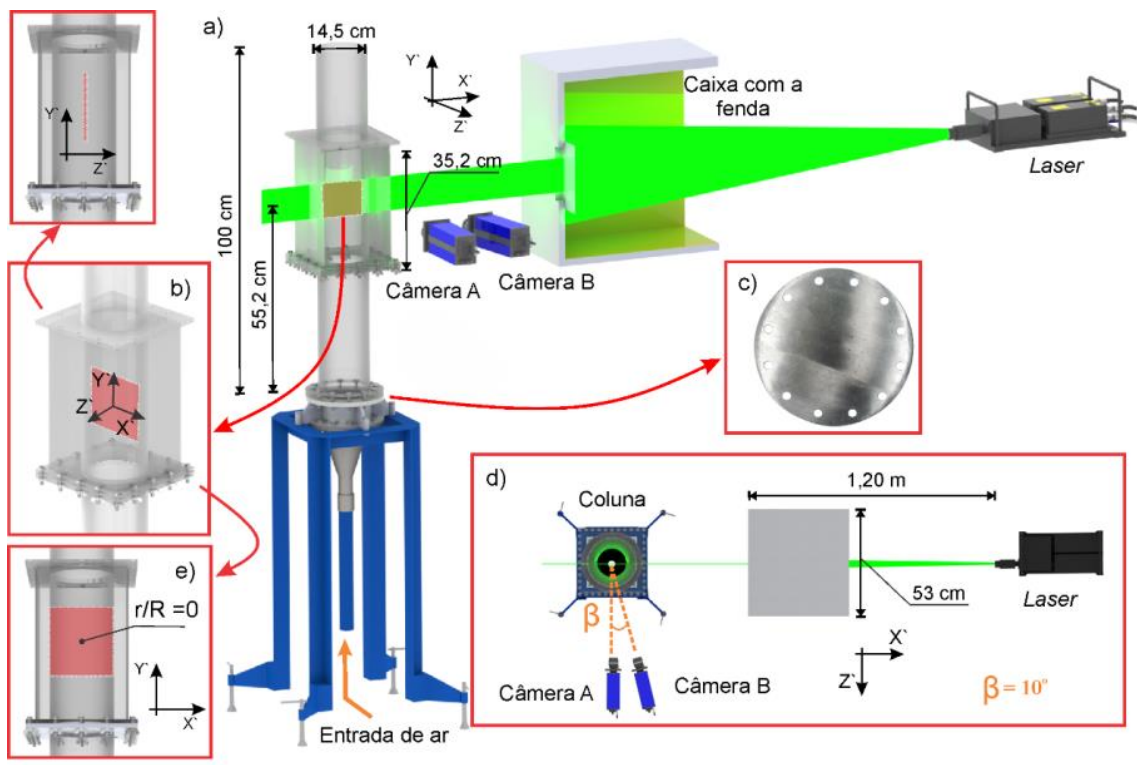

Fonte: Adaptado de AMARAL et al. (2014a). 
A área de investigação $(14,5$ x $10 \mathrm{~cm})$ estava localizada a $55,2 \mathrm{~cm}$ do distribuidor de gás. As medidas foram feitas pela câmera B (por registrar uma melhor iluminação das partículas traçadoras em relação à câmera A) utilizando a função de mapeamento com um erro de 0,96 pixel. Foram feitas medidas PIV para vazões de ar igual a 1,$5 ; 2,5 ; 5,0 ; 7,5 ; 10$; $15 ; 20 ; 30$ e $40 \mathrm{~L} / \mathrm{min}$. A vazão de ar foi controlada por um rotâmetro e suas medidas foram realizadas com um medidor digital Alicat Scientific com uma precisão de 0,1 $\mathrm{L} / \mathrm{min}$. Depois da gravação das imagens, foi feito o pré-processamento utilizando sequencialmente um filtro RMS (Rooot Mean Square) $3 \times 3$ pixels, SSM (Substract Sliding Minimum) $3 \times 3$ pixels, definindo os valores de intensidade de pixel resultante menor que $5 \mathrm{e}$ maior que 50 counts como 0 e 50 counts, respectivamente. Foi utilizada a correlação cruzada padrão aplicando a avaliação de múltiplos passos com diminuição da janela de interrogação (inicial de 256 pixels e final de 128 pixels) utilizado o Adaptive PIV. Além disso foi utilizado a sobreposição da janela de interrogação com sua vizinhança (inicial de $50 \%$ e final de $75 \%$; com passos 2 e 3). A gravação e o processamento das imagens foram feitos utilizando o software Davis 8.2.2 da LaVision. As séries temporais do módulo de velocidade foram obtidas no ponto central $\mathrm{r} / \mathrm{R}=0$ (Figura 2e). Foram gravados 4000 pares de imagens (instantâneos de campo de velocidade) com uma frequência de $4,2 \mathrm{~Hz}$ (frequência máxima do sistema PIV). O critério para a escolha de 4000 imagens foi baseado a partir do instante da não variação da média acumulada do módulo da velocidade (Figura 3). Utilizando a vazão máxima de 40 L/min (condição de maior turbulência), notase que após $400 \mathrm{~s}$ o regime encontra-se estabelecido, não havendo, portanto, a necessidade de mais aquisições de dados.

Para o cálculo da entropia de Kolmogorov foi utilizado o software RRChaos. Esse programa utiliza o método de
Schouten et al. (1994). O tempo de amostragem foi definido como o inverso da frequência de aquisição das imagens, o comprimento de corte foi defino sendo igual ao desvio padrão da série temporal do módulo de velocidade e o desvio padrão máximo no cálculo da entropia de Kolmogorov foi de $1,0 \%$.

Figura 3 - Média acumulada do módulo de velocidade para uma vazão de $40 \mathrm{~L} / \mathrm{min}$.

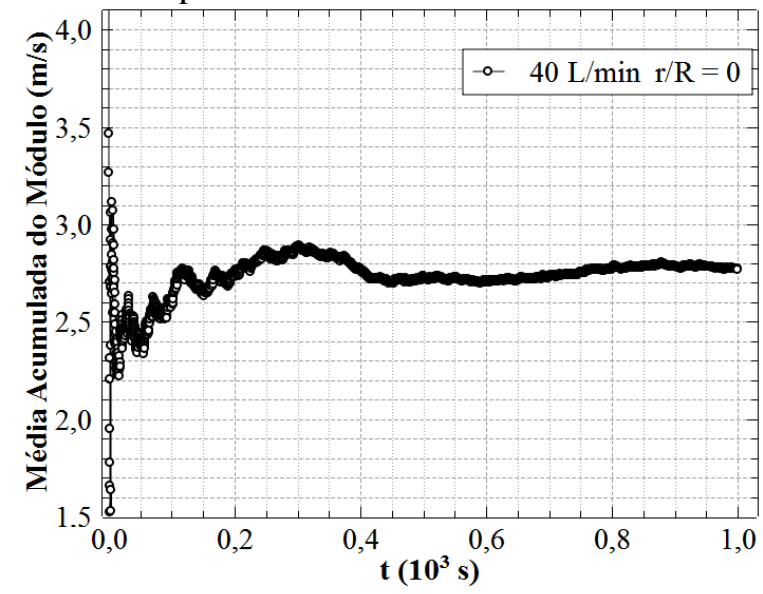

\section{RESULTADOS E DISCUSSÃO}

A Figura 4 apresenta a evolução da entropia de Kolmogorov ( $\left.\mathrm{K}_{\mathrm{ML}}\right)$ com o aumento da vazão (Q) e velocidade superficial do gás $\left(\mathrm{u}_{\mathrm{g}}\right)$.

Figura 4 - Entropia de Kolmogorov em função da vazão e velocidade superficial do gás.

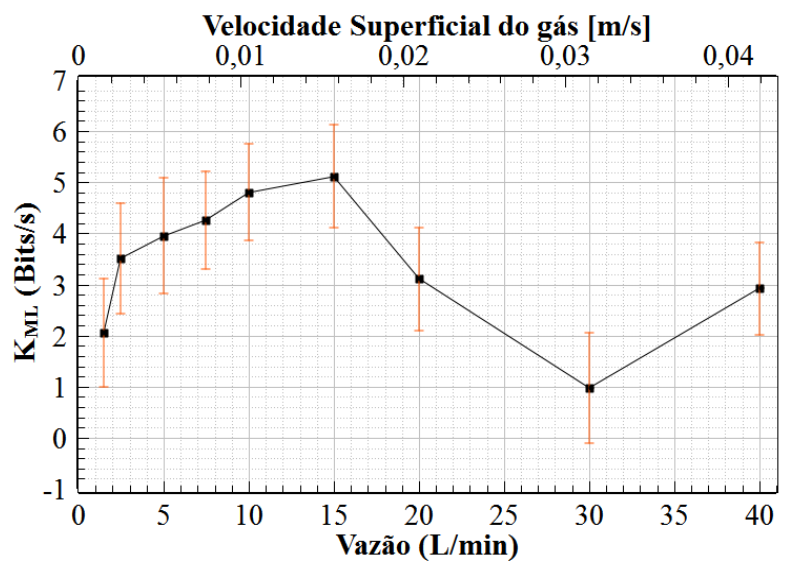


Nota-se um crescimento do valor da entropia de Kolmogorov na faixa de 1,5 - 15 L/min, sendo mais acentuado em 1,5 - 2,5 $\mathrm{L} / \mathrm{min}$. A partir da vazão $15 \mathrm{~L} / \mathrm{min}$ um decréscimo da entropia de Kolmogorov é observado até $30 \mathrm{~L} / \mathrm{min}$, voltando a aumentar o seu valor em $40 \mathrm{~L} / \mathrm{min}$.

O primeiro máximo da Figura 4, observado na vazão de $15 \mathrm{~L} / \mathrm{min}$, pode ser considerado como o regime mais complexo e de menor previsibilidade. $\mathrm{Na}$ vazão de 30 L/min é observado o primeiro mínimo indicando um comportamento com menor complexidade. Essas oscilações nos valores das entropias de Kolmogorov podem ser caracterizadas como transições de regimes. De fato, essa observação corrobora com os resultados obtidos por Nedeltchev et al. (2007, 2011). Em ambos os trabalhos, observaram-se regiões de máximos e mínimos da entropia de Kolmogorov a partir da variação da velocidade superficial do gás. Os picos de máximos da entropia de Kolmogorov foram associados como a desestabilização (instabilidades) dos estados do sistema gás líquido. Enquanto os pontos de mínimos da entropia de Kolmogorov foram considerados como estados de estabilização do sistema gás - líquido. Cada transição de regime pode ser identificada pela sequência de máximos e mínimos, isto é, em cada máximo da entropia de Kolmogorov o sistema gás - líquido apresenta uma etapa de auto-organização ao passo que em cada queda do valor deste parâmetro o sistema gás - líquido apresenta um estado de organização.

A Figura 5 apresenta os campos de velocidade do líquido (u) em função do raio da coluna para diversas vazões. Com o aumento da vazão, observa-se um aumento da velocidade da fase líquida que pode ser associado ao aumento da concentração de bolhas e, consequentemente, ao aumento de regiões de arraste. Para as vazões de 1,5 até 7,5 L/min (Figuras 5a, 5b, 5c e 5d), observase uma distribuição mais suavizada (região alvo coerente com a sua vizinhança) da velocidade média na região $0<\mathrm{r} / \mathrm{R}<1$. Contudo, para as vazões de $10-15 \mathrm{~L} / \mathrm{min}$ (Figuras 5e e 5f) é possível observar, variações mais acentuadas (distorção) dos campos de velocidade do líquido em relação às vazões menores na região $-1<\mathrm{R}<0$. Após a vazão de $15 \mathrm{~L} / \mathrm{min}$, percebe-se que as distorções aumentam em praticamente toda a área investigada, principalmente para a vazão de $40 \mathrm{~L} / \mathrm{min}$.

Figura 5 - Campos de velocidade do líquido com (a) 1,5 (b) 2,5 (c) 5,0 (d) 7,5 (e) 10,0 (f) 15,0 (g) 20,0 (h) 30,0 e (i) $40,0 \mathrm{~L} / \mathrm{min}$.

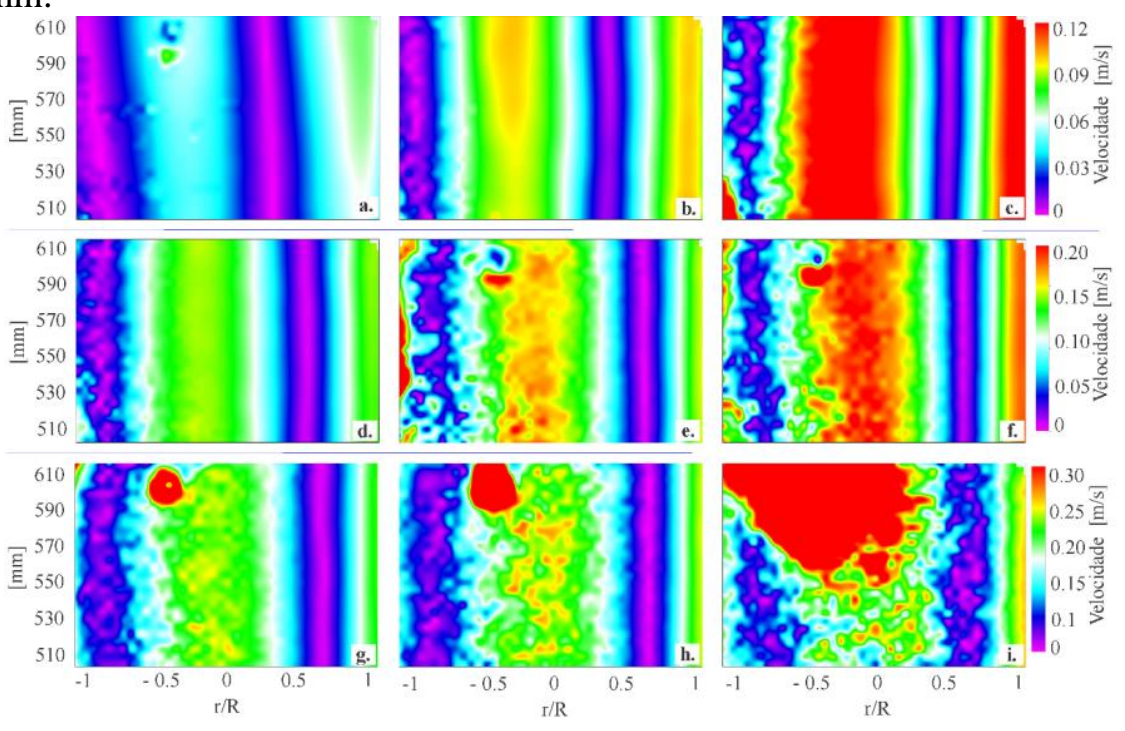


Para todas as vazões, a distribuição da velocidade médida é assimétrica. Isso pode estar relacionando ao design do distribuidor de gás, natureza do escoamento (efeito das regiões de recirculação) ou projeto da coluna. Essa distorção no campo de velocidade pode ser associada a presença das bolhas no campo de investigação. Nessa abordagem, para as vazões entre 10 e $15 \mathrm{~L} / \mathrm{min}$, as bolhas começam a se organizar homogeneamente no campo de visão. Com isso, supõe-se que o sistema gás - líquido entra em um processo de auto-organização, evidenciando uma possível região de transição. Isso corrobora com a Figura 4, em que o máximo da entropia encontra-se na vazão de $15 \mathrm{~L} / \mathrm{min}$. Nas vazões maiores que $15 \mathrm{~L} / \mathrm{min}$, a distorção pode ser vista em todo o campo de visão, relacionando assim, a inexistência de um caminho preferencial das bolhas. Relacionando com a Figura 4, o regime pode ser considerado como heterogêneo (Figuras $5 \mathrm{~g}, 5 \mathrm{~h}$ e $5 \mathrm{i}$ ) ou regime agitado (churn - turbulent). Nesse regime prevalece uma maior coalescência das bolhas e grande interação entre as fases do sistema gás - líquido. É importante ressaltar que a diminuição dos valores para entropia de Kolmogorov (Figura 4) com o aumento da vazão ou velocidade superficial do gás é justificado pela presença de uma maior recirculação do líquido e pela coalescência das bolhas.

O mesmo comportamento para esta diminuição do valor da entropia de Kolmogorov foi observado por Gourich et al. (2006) e Nedeltchev et al. (2007, 2011). A Figura 6 apresenta o perfil de vorticidade para as vazões de $1,5,5$ e $40 \mathrm{~L} / \mathrm{min}$ calculada utilizando o Ensemble Correlation para obter as melhores médias. Observa-se que de fato existe uma maior recirculação do líquido na coluna com um aumeno da vazão, em que as médias de vorticidade negativas indicam o movimento oposto ao sentido das bolhas. Essa maior recirculação do líquido pode influenciar na diminuição nos graus de liberdade do sistema, isto é, a complexidade diminui e a previsibilidade aumenta, como consequência, a entropia de Kolmogorov diminui.

Por outro lado, os altos valores da entropia em vazões baixas podem ser relacionados com $\mathrm{o}$ fato da menor homogeneidade da distribuição da velocidade do líquido, no campo de visão, causado pela região de arraste das bolhas próximas ao centro da coluna. Essa não homogeneidade da distribuição de velocidade exige uma ampla faixa de velocidade (Dynamic Velocity Range - DVR) da técnica PIV utilizada. A DVR em PIV representa a resolução espacial e temporal que a técnica é capaz de fornecer nos campos de velocidade. Com o aumento da coalescência das bolhas nas vazões de gás mais altas, a distribuição da velocidade do líquido se torna mais homogênea no campo de visão, diminuindo, portanto, o valor da entropia de Kolmogorov.

Figura 6 - Perfil de vorticidade para as vazões de $1,5,15$ e $40 \mathrm{~L} / \mathrm{min}$.

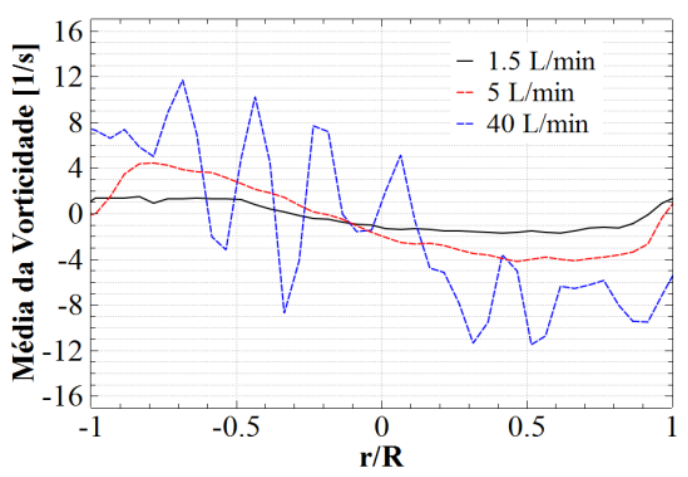

A Figura 7 apresenta a velocidade do líquido em função da vazão de gás obtida em $\mathrm{r} / \mathrm{R}=0$. Percebe-se que com o aumento da vazão, a incerteza da velocidade calculada por estatística de correlação PIV - utilizando o método de Wieneke (2014) - aumenta. Esse fato dificulta um estudo com vazões - faixa de operação - mais altas (a partir de 40 $\mathrm{L} / \mathrm{min}$ ) e, portanto, garantir a qualidade das medidas PIV. Em decorrência disso, o estado do sistema gás - líquido com uma vazão de $40 \mathrm{~L} / \mathrm{min}$ foi considerado como regime 
heterogêneo. Apesar de possuir um maior valor da entropia de Kolmogorov em relação a vazão de $30 \mathrm{~L} / \mathrm{min}$ (Figura 4) e possivelmente ser confundido como uma segunda transição de regime, não se pode afirmar com precisão se de fato ocorre alguma alteração na complexidade do sistema gás líquido próximo a vazão de $40 \mathrm{~L} / \mathrm{min}$. Isso decorre do grande número de bolhas começarem a interferir no espalhamento da luz do laser e, consequentemente, elevando o nível de ruído no campo da velocidade do líquido (Figura 5i).

Figura 7 - Velocidade e sua incerteza em função da vazão e velocidade superficial do gás.

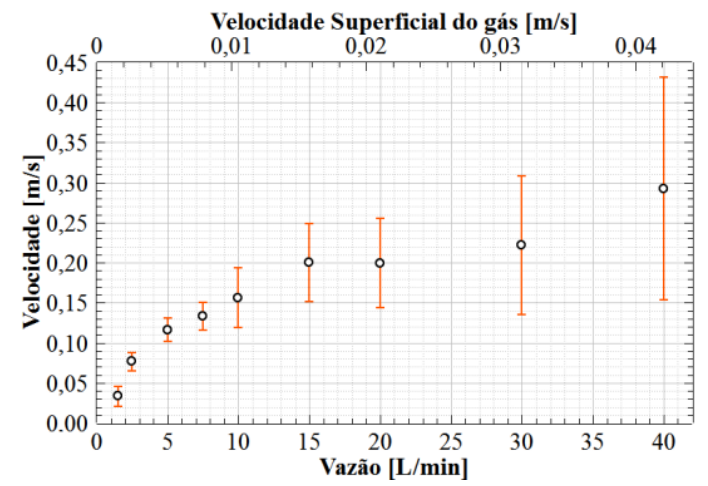

\section{CONCLUSÃO}

Esse trabalho avaliou o escoamento da fase líquida usando a análise caos a partir de medições PIV de flutuações de velocidade em uma região central de coluna de bolhas para diferentes vazões de gás. Os resultados mostram que dentro de uma faixa de operação é possível estabelecer regiões de regimes $(1,5$ - 7,5 L/min regime homogêneo; $10-15$ $\mathrm{L} / \mathrm{min}$ zona de transição e $20-40 \mathrm{~L} / \mathrm{min}$ regime heterogêneo) a partir da entropia de Kolmogorov. Apesar da baixa frequência PIV de aquisição das imagens, foi mostrado que é possível obter valores de entropia representativos. Os resultados para entropia de Komogorov apresentaram uma tendência de máximo e de mínimo, estando, portanto, de acordo com a literatura consultada. Ressalta- se que para altas vazões a técnica PIV pode gerar medidas errôneas, sendo essa a sua principal limitação.

\section{NOMENCLATURA}

$\mathrm{K}_{\mathrm{ML}}$ Entropia de Kolmogorov

[bits/s]

Q vazão

$\mathrm{u}_{\mathrm{g}} \quad$ velocidade superficial do ar

[L/min]

$[\mathrm{m} / \mathrm{s}]$

$\mathrm{u} \quad$ velocidade do líquido

$[\mathrm{m} / \mathrm{s}]$

\section{REFERÊNCIAS}

\author{
ABARBANEL, H. D. I. Analysis of \\ Observed Chaotic Data, 1996.
}

ABBASI, M.; MOSTOUFI, N.; SOTUDEHGHAREBAGH, R.; ZARGHAMI, R. A novel approach for simultaneous hydrodynamic characterization of gas-liquid and gas-solid systems. Chemical Engineering Science, v.100, p.74-82, 2013.

ADRIAN, R. Particle-imaging techniques for experimental fluid mechanics. Annual review of fluid mechanics, v. 23, p. 261-304, 1991.

AJBAR, A.; AL-MASRY, W.; ALI, E. Prediction of flow regimes transitions in bubble columns using passive acostic meansurements. Chemical Engineering and Processing: Process Intensification, v.48, p. 101-110, 2009.

AMARAL, R. L. "Uso da Velocimetria por Imagem de Partícula (PIV) na Indústria do Petróleo", Palestra UFPE, Recife, 2013.

AMARAL, R. L.; da COSTA. K. K.; FORERO D. I. S.; VIANNA, S. S. V.; TARANTO, O. P.; MORI, M. Efeito do préprocessamento de imagem PIV na velocidade do líquido em uma coluna de bolhas com expansão de diâmetro. Congresso Nacional de Engenharia Mecânica (CONEM). Uberlândia-MG, Brasil, 2014b. 
AMARAL, R. L.; FORERO D. I. S.; da COSTA. K. K.; TARANTO, O. P.; VIANNA, S. S. V.; MORI, M. Efeito da função de mapeamento na distribuição da velocidade da fase líquida na seção de uma coluna de bolhas por PIV. Congresso Brasileiro de Engenharia Química (COBEQ). Florianópolis-SC, Brasil, 2014a.

BOYER, C., D.; UQUENNE, A.M.; WILD, G. Measuring techniques in gas-liquid and gas-liquid-solid reactors. Chemical Engineering Science, v.57, p.3185-3215, 2002.

BRIENS, L.A.; ELLIS, N. Hydrodynamics of three-phase fluidized bed systems examined by statistical, fractal, chaos and wavelet analysis methods. Chemical Engineering Science, v.60, p.6094-6106, 2005.

BRÖDER, D.; SOMMERFELD, M. An advanced LIF-PLV system for analyzing the hydrodynamics in a laboratory bubble column at higher void fraction. Experiments in Fluids. v.33, p 826, 2002.

BRÖDER, D.; SOMMERFELD, M. Planar Shadow image velocimetry for the analysis of the hydrodynamics in bubble flows. Measurement Science and Technology, v.18, p. 2513-2528, 2007.

Da COSTA, K. K.; FORERO, D. I. S.; AMARAL, R. L.; TARANTO, O. P.; VIANNA, S. S. V.; MORI, M. Efeito da autocalibração para Stereo-PIV na distribuição da velocidade $2 \mathrm{D} 3 \mathrm{C}$ da fase líquida em uma seção de uma coluna de bolhas. Congresso Brasileiro de Engenharia Química (COBEQ). Florianópolis-SC, Brasil, 2014.

DECKWER, W. D., FIELD, R.W. Bubble Column Reactors. Wiley: New York, 1992.
DRAHOŠ, J.; ZAHRADNÍK, J.; PUNČOCHÁř, M.; FIALOVÁ, M.; BRADKA, F. Effect of operating conditions on the characteristics of pressure fluctuations in a bubble column. Chemical Engineering and Processing, v.29, p.107-115, 1990.

FAN, L. S. Gas-liquid-solid fluidization engineering. Stoneham, MA: Butterworth, 1989.

GOURICH, B.; VIAL, C.; ESSADKI, A.; ALLAM, F.; SOULAMI, M. B.; ZIYAD, M. Identification of flow regimes and transition points in a bubble column through analysis of differential pressure signal-Influence of the coalescence of the liquid phase, Chemical Engineering and Processing, v.45, p.214223, 2006.

GRASSBERGER, P.; PROCACCIA, I. Estimation of Kolmogorov Entropy from a Chaotic Signal, Physical Review A, v.28, p.2591-2593, 1983.

LETZEL, H. M.; SCHOUTEN, J. C.; KRISHNA, R.; VAN DEN BLEEK, C. M. Characterization of regimes and regime transitions in bubble columns by chaos analysis of pressure signals. Chemical Engineering Science, v.52, p.4447-4459, 1997.

LI, W. L.; ZHONG, W. Q.; JIN, B. S.; XIAO, R.; HE, T. T. Flow regime identification in a three-phase bubble column based on statistical, Hurst, HilbertHuang transform and Shannon entropy analysis. Chemical Engineering Science, v.102, p.474-485, 2013.

LIN, T. J.; JUANG, R. C.; CHEN, Y. C.; CHEN, C. C. Predictions of flow transitions in a bubble column by chaotic time series analysis of pressure fluctuations signals, 
Chemical Engineering Science, v.56, p.1057-1065, 2001.

MOSDORF, R.; WYSZKOWSKI, T. Experimental investigations of deterministic chaos appearance in bubbling flow. International Journal of Heat and Mass Transfer, v.54, p.5060-5069, 2011.

NEDELTCHEV, S.; JORDAN, U.; LORENZ, O.; SCHUMPE, A. Identification of various transition velocities in a bubble column based on Kolmogorov entropy. Chemical Engineering Technology, v. 30, p. 534-539, 2007.

NEDELTCHEV, S.; SHAIKH, A.; ALDAHHAN, M. Flow regime identification in a bubble column via nuclear gauge densitometry chaos analysis. Chemical Engineering Technology, v.34, p. 225-233, 2011.

PARK, S. H.; KIM, S. D. Characterization of pressure signals in a bubble column by wavelet packet transform. Korean Journal of Chemical Engineering, v.20, p.128-132, 2003.

PRASAD, A. Particle image velocimetry Review article. Current Science, v. 79, p. 5160, 2000.

SANCHEZ-FORERO D. I.; da COSTA. K. K.; AMARAL, R. L.; TARANTO, O. P.; VIANNA, S. S. V.; MORI, M. Efeito da vazão de gás na qualidade da medida da velocidade do líquido em uma seção de uma coluna de bolhas com expansão de diâmetro por PIV. Congresso Brasileiro de Engenharia Química (COBEQ). Florianópolis-SC, Brasil, 2014.

SCHOUTEN, J. C.; TAKENS, F.; VAN DEN BLEEK, C. M. Maximum-Likelihood-
Estimation of the Entropy of an Attractor. Physical Review E, v. 49, p. 126-129, 1994.

SHOU, M.C.; LEU, L.P. Energy of power spectral density function and wavelet analysis of absolute pressure fluctuation measurements in fluidized beds. Chemical Engineering Research and Design, v.83, p.478-491, 2005.

TAKENS, F. Detecting strange attractors in turbulence, In: Dynamical systems and turbulence, Lecture Notes in Mathematics, Eds. D.A. Rand e L-S. Young, Springer Verlag, v. 898, p.366-381, 1981.

VIAL, C.; CAMARASA, E.; PONCIN, S.; WILD, G.; MIDOUX, N.; BOUILLARD, J. Study of hydrodynamic behaviour in bubble columns and external loop airlift reactors though analysis of pressure fluctuations. Chemical Engineering Science, v.55, p.2957-2973, 2000.

WIENEKE, B. Generic a-posteriori uncertainty quantification for PIV vector fields by correlation statistics. 17th International Symposium on Applications of Laser Techniques to Fluid Mechanics, Lisbon, Portugal, 07-10 July, 2014.

YANG, G. Q.; DU, B.; FAN, L. S. Bubble formation and dynamics in gas-liquid-solid fluidization - A review. Chemical Engineering Science, v.62, p.2-27, 2007.

YANG, W. C. Handbook of Fluidization and Fluid-Particle Systems. CRC Press, 2003.

ZAID, F.M.; NEDELTCHEV, S.; Al DAHHAN, M. Flow Regime Identification In Fluidized Beds Based On Statistical Analysis of Both Pressure Fluctuations and Optical Probe Data. ANS Annual Meeting, Florida, 2011. 